\section{Keynote Lectures}

\section{KN-1}

\section{The vocabulary of protein-DNA interactions: Symmetry, degeneracy, modifications}

\author{
Matthias Bochtler ${ }^{1}$ \\ 1. International Institute of Molecular and Cell Biology \& Institute \\ of Biochemistry and Biophysics, Warsaw, Poland \\ email: mbochtler@iimcb.gov.pl
}

The basics of protein nucleic acid interactions have been worked out a long time ago, but new themes are still emerging. The vocabulary for the non-degenerate recognition of DNA bases is well understood, but this is not the case for semi-degenerate recognition, which may occur "accidentally", or be imposed by symmetry. In the first part of my talk, I will focus on principles of semi-degenerate readout of DNA sequence, by minor groove readout, base unstacking, and nucleotide flipping (1). In the second part of my talk, I will concentrate on modification specific DNA binding. How nucleic acid modifications such as methylation inhibit DNA binding by steric exclusion is readily understandable. Whether a predicted clash is biologically relevant depends on whether protein and nucleic acid are sufficiently flexible to avoid the steric conflict (2). How nucleic acid conformations become a prerequisite for binding is harder to understand, especially when the dependence on the modification is stringent and when a modifier group (such as a methyl group) offers little opportunity for strong attractive interactions. In my talk, I will focus on several published and unpublished structures from our own work which illustrate the modification selective binding of nucleic acids containing 6-methyladenine, 5-methylcytosine $(5 \mathrm{mC})$, 5-hydroxymethylcytosine $(5 \mathrm{hmC})$, and glucosyl-5-hydroxymethylcytosine (g5hmC) (3).

References:

(1) Bochtler, M., Szczepanowski, R.H., Tamulaitis, G., Grazulis, S., Czapinska, H., Manakova, E. and Siksnys, V. (2006) Nucleotide flips determine the specificity of the Ecl18kI restriction endonuclease. EMBO J, 25, 2219-2229.

(2) Mierzejewska, K., Bochtler, M., Czapinska, H. (2016) On the role of steric clashes in methylation control of restriction endonuclease activity. Nucleic Acids Res, 44, 485-495.

(3) Mierzejewska, K., Siwek, W., Czapinska, H., Kaus-Drobek, M., Radlinska, M., Skowronek, K., Bujnicki, J.M., Dadlez, M. and Bochtler, M. (2014) Structural basis of the methylation specificity of R.DpnI. Nucleic Acids Res, 42, 8745-8754.

Keywords: Symmetry, degeneracy, modifications
$\mathrm{KN}-2$

\section{The Joy of Seeing - in honour of Dorothy Hodgkin}

Eleanor Dodson ${ }^{1}$

1. University of York, York, United Kingdom

email: eleanor.dodson@york.ac.uk

To quote Dorothy Hodgkin: The great advantage of X-ray analysis as a method of chemical structure analysis is its power to show some totally unexpected and surprising structure and to do so with absolute certainty.

I will show examples of how Fourier analysis using X-ray observations from a crystal, combined with imperfect phases, can allow us to boot-strap our way to a satisfactory structure. Combining these basic principals with the great computing power, and excellent data acquisition facilities now available has extended our power to "see" to new levels.

\section{References:}

[1] Hodgkin, D. C. Pickworth, J. Robertson, J. H. Trueblood, K. N. Prosen, R. J. \& White, J. G. (1955). Nature, 176 (4477), 325-328.

[2] Jenkins, H. T. (2018). Acta crystallographica. Section D, Structural biology, 205-214.

[3] Rodríguez, D. D. Grosse, C. Himmel, S. González, C. de Ilarduya, I. M. Becker, S. Sheldrick, G. M. \& Usón, I. (2009) Nature Methods, 6(9), 651-653.

Keywords: Fourier analysis, computing techniques 